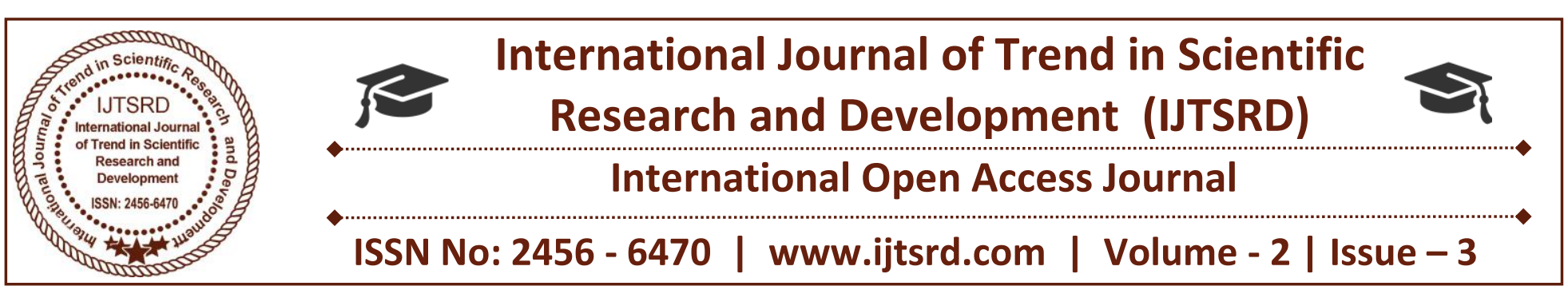

\title{
Social Group Recommendation based on Big Data
}

\author{
Ms. Nikita S. Mohite, Mr. H. P. Khandagale \\ Computer Science and Technology, Department of Technology, \\ Shivaji University, Kolhapur, Maharashtra, India
}

\begin{abstract}
\end{abstract}
Current life involves physical enjoyment, social activities and content, profile and cyber resources. Now it is easy to merge computing, networking and society with physical systems to create new revolutionary science, technical capabilities and better quality of life. That all possible through Cyber Physical Social Content and Profile Based System (CPSCPs).In this propose system, a group-centric intelligent recommender system named as GroRec, which integrates social, mobile and big data technologies to provide effective, objective and accurate recommendation services. This provides group recommendation in CPSCPs domain. In which activity oriented cluster discovery, the revision of rating information for improved accuracy and cluster preferences modelling that supports descent context mining from multiple sources. Group recommendation is based on profile and content based approach. Our main goal is make several interactions with group members by using specific technique and methods. The recommender system is economical, objective and correct.

Keywords: Big data, Data mining, Big data analysis, Recommended services, CPSCPs

\section{INTRODUCTION}

Now a day's peoples are mostly connected with Internet of things, this help to interact with whole world. Ios use for network connectivity which enables objects to connect and exchange data. A social network services (SNS) is online platform that people use to build social networks with other people who share similar personal or career interests, activities, background or real life connection. In particular, through advanced mobile and sensing technologies, social data can easily be collected as records of social events, which can be analysed to yield a better understanding of users' daily activities, social circles, living habits, etc.

CPSS is a kind of common complex system that is constituted by physical system, and its social system including human beings, and cyber system that connects both of them with each other. CPSS provide the individual centric approach it is difficult to provide Ulifusers with timely and suitable recommendations.

In our proposed system CPSCPs (Cyber-PhysicalSocial-Content-Profile based systems) is major objective. It involves Cyber components are about the computing, communication, and controls that are discrete, logical, and switched. Physical components are about natural and human-made physical systems, which are governed by the laws of physics and operated in continuous time. Physical based system includes the place and time of the user. Social components include the daily activities of users, communication network with others. User profile became central feature of social networking sites, allowing users to compile list of "friends" and search for other users with similar interest. In content based system content based filtering is used. The information source that content based systems are mostly used with words which are occurred in text documents. Content based filtering recommends items based on comparison between the content of the items and user profile.

In social network system used discontinuous nature of data collection due to this sparsity problem is occurred. To address this challenge we propose 
group-centric intelligent recommender system in which multi-dimensional resources are invoked. The system collects high dimensional data from user's profile, groups are recommended based on the similarity of user behavior.

\section{LITERATURE SURVEY}

"Data Mining for the Internet of Things :-Literature Review and Challenges ", Feng Chen, Pan Deng, Jiafu Wan, Daqiang Zhang, Athanasios V. Vasilakos and Xiaohui Rong [5] ,in this paper, they reviewed data mining in knowledge view, technique view, and application view, including classification, clustering, association analysis, time series analysis and outlier analysis.

"Cyber-physical-social System in Intelligent Transportation”, G. Xiong, F. Zhu, X. Liu, X. Dong, W. Huang, S. Chen, and K. Zhao[11],in this paper, they closely analysed an artificial system, computational experiments and parallel execution (ACP) methodology is introduced based on which data-driven models are applied to social system. The ACP proven effective framework and scientific foundation. The result of CPSS and ACP are applied and verified in traffic areas such as management plan optimization, large scale evacuation and public traffic scheduling, manufacturing and transformation. There are achievements in both research and application in 2010 a new department, CPSS is founded in IEEE "Intelligent Systems" and "CPSS based Transportation".

"A CPSS Approach for Emergency Evacuation in Building Fires", Y. Hu, F.-Y. Wang, and X. Liu [12], in this they propose a cyber- physical-social systems (CPSS) approach based (ACP) methodology using artificial systems, computational experiments, and parallel execution. CPSS provides a tool to verify or evaluate emergency evacuation plan. It used in the significantly improve fire emergency management and public safety in building fire. CPSS is one which integrate with human and social characteristics and bridge the physical world, cyberspace, and human society together. It exists in newly rising network society "A group recommendation system with consideration of interactions among group members", Yen-Liang Chen, Li-Chen Cheng, Ching-Nan Chuang, [14], in this paper, they closely reviewed group recommendation system. The system is designed based on the framework of collaborative filtering for this they especially use genetic algorithm to predict the possible interactions among group members so that we can correctly estimate the rating that a group of members might give to an item. The experimental results show that the proposed system can give satisfactory and high quality group recommendations. This group recommendation system applied in online store sales and are effective way to increase customer satisfaction and consequently customer loyalty.

\section{NEED OF WORK}

Social-Network-services (SNS) is a web based service that allowed individuals to connect with public or semi-public profile within bounded system. In SNS users created profile with specific content pattern. The purpose of this system is to communication with social media and share users post. So the SNS platform is used in the CPSS.

Recently, the physical system and its cyber system of CPS are designed, built and used by human beings in their social and natural environments, the social system must be of the same importance as its CPS; these indivisible three parts (cyber, physical, and social system) constitute the entity named as cyberphysical-social system (CPSS).Physical \& social system and their cyber systems can understand each other, their stepwise interaction helps improve each other, and gradually the effective control and safe, reliable and efficient operation of CPSS will be realized. This can improve the perception, analysis and control ability of those complex CPSSs, and their effective control and the safe, reliable and efficient operation can be achieved. CPSS to achieve the reliable and efficient operation and optimization target in normal situation.

SNS and CPSS integrated into our proposed system named as CPSCPs (Cyber-Physical-Social-ContentProfile based system) in which content based system content is going to be refer and profile is refer in profile based system. 
IV. OUTLINE OF PROPOSED WORK

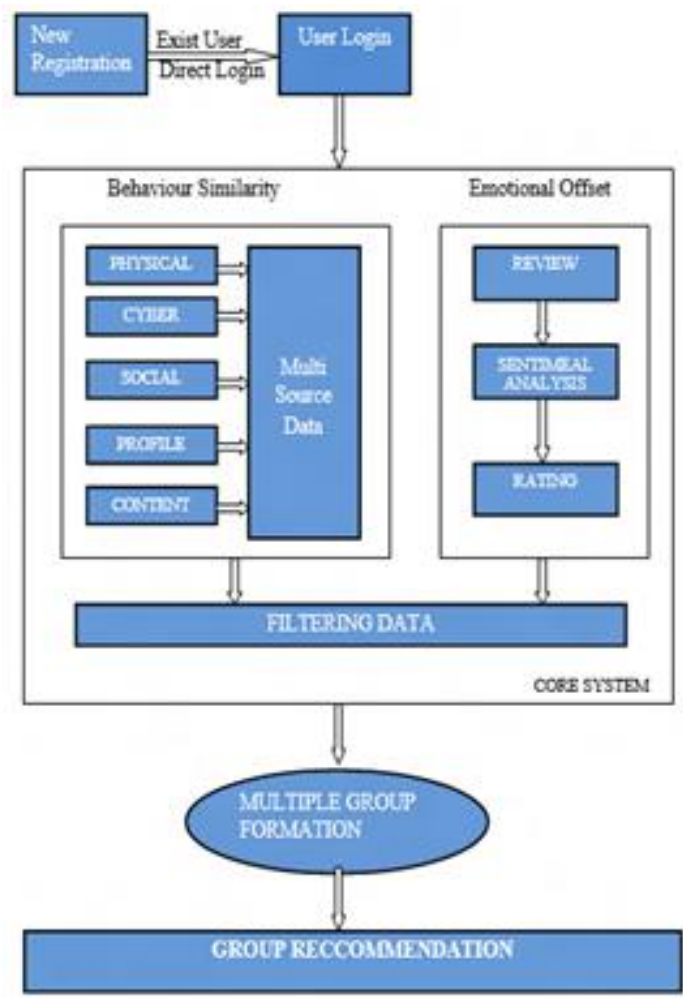

Fig : Architecture Diagram of Proposed Work

The proposed system will be consist of 6 modules:-

1. Login Module:-

Login module is a web application where user can login. If user is existing user then they can login with the help of user-id and password. If user don't have the authentication then user click on new user registration.

\section{New User Registration:-}

It is necessary to register the new user and get the user name and password for login. In this registration form person fill the all personal information like name, DOB, age, current location, school name, etc.

\section{Core Module:-}

Core module consists of Big data for efficient storage of multidimensional data. Big data is a term used for a collection of data sets so large and complex that it is difficult to process using traditional applications/tools. The unstructured data can be structured by using Big data technology.

\subsection{Group Discovery supported behavioural Similarity:-}

In Group Recommendation, the cluster discovery module is meant to speedily and accurately discover teams from high-dimensional information supported the similarity of user behaviour.

\subsection{Method of Rating Revision supported Emotional Offset selection through LDA:-}

In Group Recommendation, rating information are revised in accordance with their emotional options that are extracted from user reviews through sentiment analysis. Considering that user reviews will embrace a major emotional offset, sentiment analysis is a crucial suggests that of calculative this offset to revise the initial ratings. The rating revision module is that the foundation for guaranteeing the objectiveness of the recommender, thereby raising the accuracy of the advice results. LDA (Latent Diritchlet Allocation) is used in sentimental analysis for finding the topic from posts. While doing the sentimental analysis the topic searching part from user reviews are done. This will help for the formation of group based on reviews.

\section{Filtering Of Data:-}

In the filtering of data format we filter all cyberphysical-social-content-profile and make in standard format with similarities and store again on our database for making the group with similar data. For this purpose $\mathrm{C}$ we will use $\mathrm{KNN}$, K-means, collaborative filtering.

\section{Multiple Group Formation:-}

To making the group on the basis of behavioural and content base using rollup and cube for similar properties and similar attributes of people.

\section{Group Recommendation:-}

Whenever new user login then their activities are matched with our existing groups. Then our system will be sending recommendation for that person to add it.

\section{CONCLUSION}

SNS provides individual centric recommendation, this make complex, non-efficient system. So to address this problem we comes with Group centric recommendation based on CPSCPs. This provide effective, objective and accurate recommendation services in CPSCPs. Our proposed system provides group recommendation system based on behavior similarity and emotional offset among users. This help to reduce complexity of conventional individual centric recommender systems. The emotional offset extracted from reviews, sentiment analysis is 
proposed to revise user rating for improved result of rating data. Cluster data is achieve by applying $\mathrm{KNN}$, K-means, collaborative filtering, content based filtering. This group recommendation system gives efficient, effective and accurate results.

\section{REFERENCES}

1. Alexander W. Schneider and Boltzmannstr, "Using Web Analytics Data to support Social Software Users", 2010, srvmatthes5.in.tum.de.

2. Gautam Shroff, Lipika Dey and Puneet Agrawal, "Social Business Intelligence Using Big Data", 2013.

3. V. Jude Nirmal and D.I. George Amalarethinam, "Parallel Implementation of Big Data PreProcessing Algorithms for Sentiment Analysis of Social Networking Data", IJFMA Vol. 6, No. 2, 2015, 149-159, ISSN: $2320-3242$ (P), $2320-$ 3250 (online) Published on22 January 2015.

4. R. Balaji Ganesh \& S. Appavu, "An Intelligent Video Surveillance Framework with Big Data Management for Indian Road Traffic System", IJCA (0975 - 8887) Volume 123 - No.10, August 2015.

5. Feng Chen, Pan Deng, Jiafu Wan, Daqiang Zhang, Athanasios V. Vasilakos and Xiaohui Rong, "Data Mining for the Internet of Things: Literature Review and Challenges", Hindawi Publishing Corporation, International Journal of Distributed Sensor Networks, Article ID 431047, March 2015.

6. Somesh S Chavadi and Dr. Asha T, "Text Mining Approach for Big Data Analysis Using Clustering and Classification Methodologies", IJETAE, Volume 4, Issue 8, August 2014.

7. Catherine Dwyer, Yi Zhang and Starr Roxanne Hiltz, "Using Web Analytics to Measure the Activity in a Research-Oriented Online Community, Paper Submit to the Tenth Americas Conference on Information Systems", New York, , August 2004, pp 2668-78.

8. Sana Siddiqui and Imran Qadri, "Mining Web Log Files for Web Analytics and Usage Patterns to Improve Web Organization", Siddiqui et al., IJARCSSE 4(6), June - 2014, pp. 794-802.

9. Vatsal Shah, "Big Video Data Analytics using Hadoop”, IJARCSSE, Vol 5, issue 7, July 2015.
10. F.-Y. Wang, "The emergence of intelligent enterprises: From CPS to CPSS," Intelligent Systems, IEEE, vol. 25, no. 4, pp. 85-88, 2010.

11. G. Xiong, F. Zhu, X. Liu, X. Dong, W. Huang, S. Chen, and K. Zhao, "Cyber-physical-social system in intelligent transportation," Auto-matica Sinica, IEEE/CAA Journal of, vol. 2, no. 3, pp. 320-333, 2015.

12. Y. Hu, F.-Y. Wang, and X. Liu, "A CPSS approach for emergency evacuation in building fires," IEEE Intelligent Systems, no. 3, pp. 48-52, 2014.

13. A. Sheth, P. Anantharam, and C. Henson, "Physical-cyber-social computing: An early 21st century approach," Intelligent Systems, IEEE, vol. 28, no. 1, pp. 78-82, 2013.

14. Y.-L. Chen, L.-C. Cheng, and C.-N. Chuang, "A group recommendation system with consideration of interactions among group members," Expert systems with applications, vol. 34, no. 3, pp. 2082-2090, 2008.

15. H. Ning, H. Liu, J. Ma, L. T. Yang, and R. Huang, "Cybermatics: Cyber-physical-social-thinking hyperspace based science and technology," Future Generate. Compute. System, 2015. 